\title{
Dopant Diffusion under Pressure and Stress
}

\section{Citation}

Aziz, Michael J. 2003. Dopant diffusion under pressure and stress. In Proceedings of the IEEE International Conference on Simulation of Semiconductor Processes and Devices SISPAD 2003: Boston, Massachusetts, 3-5 September 2003, 137-142. Piscataway, N.J.: IEEE.

\section{Published Version}

http://dx.doi.org/10.1109/SISPAD.2003.1233656

\section{Permanent link}

http://nrs.harvard.edu/urn-3:HUL.InstRepos:2961881

\section{Terms of Use}

This article was downloaded from Harvard University's DASH repository, and is made available under the terms and conditions applicable to Other Posted Material, as set forth at http:// nrs.harvard.edu/urn-3:HUL.InstRepos:dash.current.terms-of-use\#LAA

\section{Share Your Story}

The Harvard community has made this article openly available.

Please share how this access benefits you. Submit a story.

Accessibility 


\title{
Proceedings of 2003 International Conference on Simulation of Semiconductor Processes and Devices, Cambridge MA, September 2003 Dopant Diffusion under Pressure and Stress
}

\author{
Michael J. Aziz \\ Division of Engineering and Applied Sciences \\ Harvard University \\ Cambridge, MA USA \\ maziz@harvard.edu
}

\begin{abstract}
The effects of stress on equilibrium point defect populations and on dopant diffusion in strained semiconductors are reviewed. The thermodynamic relationships presented permit the direct comparison of hydrostatic and biaxial stress experiments and of atomistic calculations of defect volumetrics for any proposed mechanism. Experiments on the effects of pressure and stress on the diffusivity of $B$ and $\mathrm{Sb}$ are reviewed. The opposite effects of hydrostatic compression and of biaxial compression on the diffusivity are a consequence of the non-local nature of the point defect formation volume. Comparisons between these effects are made to determine quantitatively the anisotropy of the migration volume. The requirements to permit the prediction of the effect of an arbitrary stress state on diffusion in an arbitrary direction are discussed.
\end{abstract}

Keywords-diffusion; dopant; stress; point defects; pressure

\section{INTRODUCTION}

Because understanding and controlling diffusion-related phenomena become increasingly important as semiconductor device dimensions decrease, atomic diffusion in $\mathrm{Si}$ has been heavily studied [1]. The study of stress effects on diffusion, which have been characterized experimentally starting a decade ago [2-5], is important for understanding the processing and stability of strained Si and Si-Ge films. Additionally, although bulk wafers cannot sustain significant nonhydrostatic stresses at diffusion temperatures, such stresses are sustained near interfaces with patterned films and in the films themselves. These stresses can be quite large due to growth stresses, interfacial stresses, thermal expansion mismatch, or dislocations [6]. The complexities associated with nonhydrostatic stress states in patterned materials (as well as in initially biaxially strained planar films after the breakdown of a smooth, flat film morphology) make the interpretation of stress effects in terms of basic mechanisms and the prediction of stress effects from known mechanisms quite difficult. However, in certain cases, a measurement of the diffusivity under hydrostatic pressure and simple nonhydrostatic stress states can provide sufficient information to permit the prediction of behavior under arbitrary stress states [7-12].

The atomic diffusivity in a material with cubic crystallography is given by

$$
D=\frac{1}{6} \lambda^{2} \vee f \exp \left(\frac{-G^{*}}{k_{B} T}\right)
$$

This research was supported by NSF-DMR-0213373 and NSF-DMR-9813803

$$
G^{*}=G f_{+} G^{m}
$$

where $\lambda$ is the jump distance, $v$ is an effective vibrational frequency [13], $G^{*}$ is the Gibbs free energy of activation, $G f$ is the Gibbs free energy of formation of the mobile species, $G^{m}$ is the Gibbs free energy of migration of the mobile species, and departures from a true random-walk are accounted for by the correlation factor $f$. For example, for diffusion of an impurity "A" by a normal vacancy mechanism, the mobile species (which is called an A-V complex) is A on a lattice site bound to a vacancy, and $G f$ is the change in Gibbs free energy when one vacancy is created at a kink site on a step at the surface and placed next to the impurity in the lowest free energy configuration. The free energy of migration $G^{m}$ is the change in free energy when the mobile species moves to the saddle point of its migration path.

\section{DIFFUSION UNDER STRESS}

To understand the various influences of pressure and stress on the diffusivity $[7,14]$, one must consider both the effects on the point defect concentrations $[6,7,15]$ and on the point defect mobilities [11,16,17].

The thermodynamics of point defect formation in heavily dislocated crystals under hydrostatic or nonhydrostatic stress has been well understood for decades. When experimental conditions are such that the point defect concentrations equilibrate with sources and sinks rapidly compared to the experimental time scale, then the pressure-dependence of the point defect concentration is characterized by the point defect formation volume $V^{f}$, a scalar. The new situation that requires the assignment of tensor properties to the formation volume is that large single crystals entirely free of extended defects, at which point defects can internally equilibrate, are now used. Consequently, in a single crystal free of internal point defect sources, the volume change upon point defect formation does not tell the entire story: the crystal changes shape, the shape change depends upon which surface(s) are sources/sinks for defect equilibration ${ }^{1}$, and stresses interact with the components

\footnotetext{
${ }^{1}$ For example, the thermal expansion coefficient of a thin wafer free of extended defects is predicted to be greater in the thin direction than in the orthogonal directions because most of the thermally injected point defects come from the thin direction [8].
} 
of that shape change in the characteristic stress : strain tensor manner.

Let us focus on the diffusion coefficient in the direction normal to an (001) surface, which is the direction measured in practically all experiments, and let the dopant diffusivity in this direction be $D_{33}(T, \boldsymbol{\sigma})$, where $\boldsymbol{\sigma}$ is the stress tensor. The effect of stress on $D_{33}$ is characterized by the activation strain tensor [7] $\mathbf{V} *$ with components

$$
V_{i j}^{*}=k_{B} T\left(\frac{\partial \ln D_{33}}{\partial \sigma_{i j}}\right)
$$

Under hydrostatic pressure $\mathbf{D}$ is isotropic and $\sigma_{11}=\sigma_{22}=\sigma_{33}=$ $-p$. The pressure effect is then minus the sum of these three uniaxial effects. In this case the pressure effect is characterized by scalar activation volume $V^{*}$ :

$$
V^{*}=-k_{B} T\left(\frac{\partial \ln D}{\partial p}\right)
$$

$V^{*}$ is therefore the sum of the three diagonal elements of the activation strain tensor ${ }^{2}$. The activation volume can also be written as the pressure-derivative of the Gibbs free energy of activation:

$$
V^{*}=\left(\frac{\partial G^{*}}{\partial p}\right)
$$

We can interpret (5) to mean that pressure affects the diffusivity through a Boltzmann factor in the change in $G^{*}$ in (1), where the change is due to the $p V$ work done by the pressure medium against the volume changes associated with defect formation and migration ${ }^{3}$. These changes are shown schematically in Fig. 1.

Under nonhydrostatic stress, the Gibbs free energy is difficult to define, but the stress-induced change in diffusivity can still be written as a Boltzmann factor in the work done by the interaction of the activation strain tensor and the stress state [17] as indicated below. As the activation strain tensor is the sum of the formation strain tensor and the migration strain tensor, let us consider them separately.

\footnotetext{
2 To encompass cases in which several mechanisms contribute significantly to transport, (3) and (4) are more properly interpreted as the apparent activation strain tensor and volume arising from the weighted contributions of those several mechanisms. In the ensuing discussion we assume a single mechanism for simplicity.

3 Neglecting variations in $v$ and $\lambda$ is practically always justified, but the pressure-dependence of $f$ may or may not be negligible, depending on whether there is a significant pressure-dependence to the defect-impurity interaction. This pressure effect is analogous to the additional term in the expression for the apparent activation enthalpy introduced by a significantly temperaturedependent binding, which has been analyzed by $\mathrm{Hu}$ [22].
}

For point defects formed at the (001) surface, the dimension changes of the crystal upon point defect formation can be described by the formation strain tensor $\mathbf{V}^{\mathbf{f}}$ :

$$
\mathbf{V}^{f}= \pm \Omega\left[\begin{array}{lll}
0 & & \\
& 0 & \\
& & 1
\end{array}\right]+\frac{V^{r}}{3}\left[\begin{array}{lll}
1 & & \\
& 1 & \\
& & 1
\end{array}\right]
$$

The + sign is for vacancy formation and the - sign is for interstitial formation throughout this paper. $\Omega$ is the atomic volume, representing the dimension changes of the crystal upon creation or annihilation of a lattice site, before permitting relaxation of the lattice around the newly-created point defect. The relaxation volume $V^{r}$ propagates out elastically to all of the sample surfaces and provides equal contributions along all axes, on average, after point defect equilibration throughout the sample volume. The dependence of the equilibrium defect concentration $C^{e}$ on applied stress $\sigma$ can be written

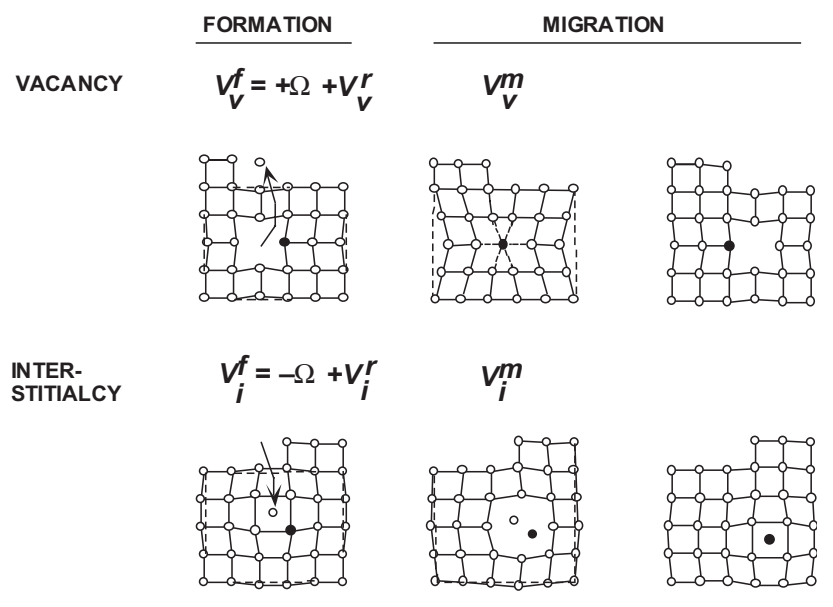

Figure 1. Cartoon of formation and migration volumes for vacancy (top) and interstitialcy (bottom) diffusion mechanisms. Saddle point in migration path is center column. $P V^{f}$ and $P V^{m}$ interaction with externally applied pressure determines pressure-dependence of equilibrium point defect concentrations and mobilities, respectively. If $V^{f}$ for vacancies is positive and $V^{m}$ for vacancies is negative, as depicted at top, then increasing pressure squeezes out vacancies (raises $G^{f}=E^{f}-T S^{f}+P V^{f}$ ) and increases their mobility (reduces $\left.G^{m}=E^{m}-T S^{m}+P V^{m}\right)$. Tensor character of volumes is evident from the work done against a nonhydrostatic stress state during the processes depicted.

$$
\frac{C^{e}(\boldsymbol{\sigma})}{C^{e}(\mathbf{0})}=\exp \left(\frac{\boldsymbol{\sigma}: \mathbf{V}^{\mathbf{f}}}{k_{B} T}\right)
$$

where $\mathbf{V}^{\mathbf{f}}$ has the elements 0 and 1 placed appropriately in (6) for the surface at which the defects equilibrate, and the colon indicates the inner product $\sum_{i, j=1}^{3} \sigma_{i j} V_{i j}^{f}$. These dimension changes and their interactions with nonhydrostatic stresses are indicated schematically for a simple case in Fig. 2. 
Phenomenologically, we expect that the defect mobility $M$ in directions parallel and perpendicular to the direction of applied stress may differ $[11,16,17]$. $D_{33}$ depends on $M_{33}$, the mobility normal to the (001) surface. The effect of stress on M33 is characterized by the migration strain tensor, which is expected to have the form

$$
\mathbf{V}_{33}^{\mathbf{m}}=\left[\begin{array}{ccc}
V_{\perp}^{m} & & \\
& V_{\perp}^{m} & \\
& & V_{\|}^{m}
\end{array}\right] .
$$

$V_{\perp}^{m}$ and $V_{\|}^{m}$, respectively, are the dimension changes of the crystal parallel and perpendicular to the direction of net transport when the point defect reaches its saddle point. As the diffusivity is proportional to the product of the concentration and mobility of defects, the effect of stress on the diffusivity in the direction normal to the surface is obtained by the combination of (6) and (8):

$$
\begin{aligned}
\frac{D_{33}(\sigma)}{D_{33}(0)} & =\exp \left(\frac{\boldsymbol{\sigma}: \mathbf{V}_{33}^{*}}{k_{B} T}\right) ; \\
\mathbf{V}_{33}^{*} & =\mathbf{V}^{f}+\mathbf{V}_{33}^{m} .
\end{aligned}
$$

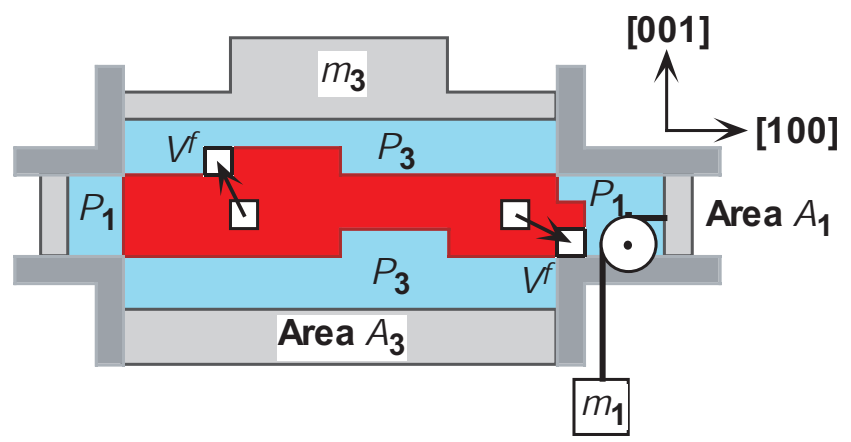

Figure 2. Non-local vacancy formation strain tensor. Pressures $P_{1}$, and $P_{3}$, of incompressible fluids (blue) in contact with (100) and (001) faces, are maintained by pistons driven by masses $m_{1}$ and $m_{3}$. Crystal volume changes by $V^{f}$ upon vacancy formation at (100) or (001) surface but work done against gravity differs $\left(m g \Delta h=P V^{f}\right)$ causing different point defect concentrations in local equilibrium with (100) and (001) surfaces.

Hydrostatic pressure then influences $D_{33}$ according to

$$
\frac{D_{33}(p)}{D_{33}(0)}=\exp \left(\frac{-p\left[ \pm \Omega+V^{r}+V^{m}\right]}{k_{B} T}\right),
$$

where the sum of the bracketed terms in (11) is the conventionally-defined scalar activation volume, and the conventionally-defined scalar migration volume $V^{m}$ is the trace of (8). Biaxial stress influences $D_{33}$ according to

$$
\frac{D_{33}\left(\sigma_{\text {biax }}\right)}{D_{33}(0)}=\exp \left(\frac{\sigma_{\text {biax }}\left[\frac{2}{3} V^{r}+V^{m}-V_{\|}^{m}\right]}{k_{B} T}\right) .
$$

\section{EXPERIMENTAL RESULTS}

We have used a clean and hydrostatic liquid Ar pressure medium and measured a pressure-enhanced diffusivity of B [9] in $\mathrm{Si}$, characterized by an activation volume of $V^{*}=-0.17 \Omega$, and a pressure-retarded diffusivity of $\mathrm{Sb}$ [10] in $\mathrm{Si}$, characterized by an activation volume of $V^{*}=+0.07 \Omega$. Note that these values are much smaller in magnitude than one atomic volume, and that such a small pressure effect implies from (11) that $V^{r}+V^{m}$ is large enough in magnitude to nearly offset the $\pm \Omega$ term. Hence we should expect the stress effect in (12) to be relatively large.

The influence of biaxial stress on the diffusivity has been characterized experimentally [2-5] by the apparent change in activation energy with biaxial (tensile) strain, $\varepsilon_{\text {biax }}$, at constant composition:

$$
Q^{\prime} \equiv-k_{B} T \frac{\partial \ln D_{33}}{\partial \varepsilon_{\text {biax }}}
$$

By comparison to (13), the combination of volumes in square brackets in (12) is equal to $-Q^{\prime} / Y$, where the biaxial modulus $Y$ is the ratio of Young's modulus to one minus Poisson's ratio. Additionally, one must take care to isolate experimentally the stress effect from the composition effect; this has been done in some of the most recent experiments [3-5].

To predict the hydrostatic or biaxial stress effects with Eqs. (11) or (12), respectively, the three parameters $V^{r}, V_{\perp}^{m}$ and $V_{\|}^{m}$ must be known. However, certain combinations of experimental observables are independent of some of the parameter values. Combining (8) and (11)-(13) results in

$$
V^{*}+\frac{3}{2} \frac{Q^{\prime}}{Y}= \pm \Omega+\left(V_{\|}^{m}-V_{\perp}^{m}\right) .
$$

If we assume that the anisotropy in the migration strain tensor (the "migration strain anisotropy" or "migration volume anisotropy") is negligible, then the right-hand side of (14) should be $+1 \Omega$ for a vacancy mechanism and $-1 \Omega$ for an interstitial-based mechanism. It turns out that there are many cases where it has been argued that crystal symmetry dictates zero migration strain anisotropy.

The cases of $\mathrm{Sb}$ and $\mathrm{B}$ diffusion in $\mathrm{Si}$ are particularly simple because there is a growing consensus that the former 
diffuses almost entirely by a vacancy mechanism and the latter diffuses almost entirely by an interstitial-based mechanism [18]. For the simple vacancy mechanism the anisotropy in the macroscopically-measured migration strain in (001) wafers should be zero due to crystal symmetry [7]. For boron diffusion by the interstitialcy mechanism in (001) wafers Daw and coworkers $[11,12]$ predict that the migration strain anisotropy is zero, due to a detailed consideration of the effects of strain on the 768 migration paths of the Boron-selfinterstitial pair that are energetically degenerate in unstrained material. My own interpretation of their result is that zero migration strain anisotropy is a consequence of having a high enough symmetry that there is a sufficient degeneracy of diffusion pathways. A particular stress state then reduces the barrier to some of them and raises the barrier to others. A symmetry-equivalent stress state (e.g. nonzero $\sigma_{33}$ with other elements zero instead of a nonzero $\sigma_{11}$ with other elements zero), however, has the same effect on a symmetry-equivalent set of migration barriers. Interestingly, they predict a significant migration strain anisotropy for vertical boron diffusion in (111) wafers. The minimal set of conditions sufficient for zero migration strain anisotropy is currently not known.

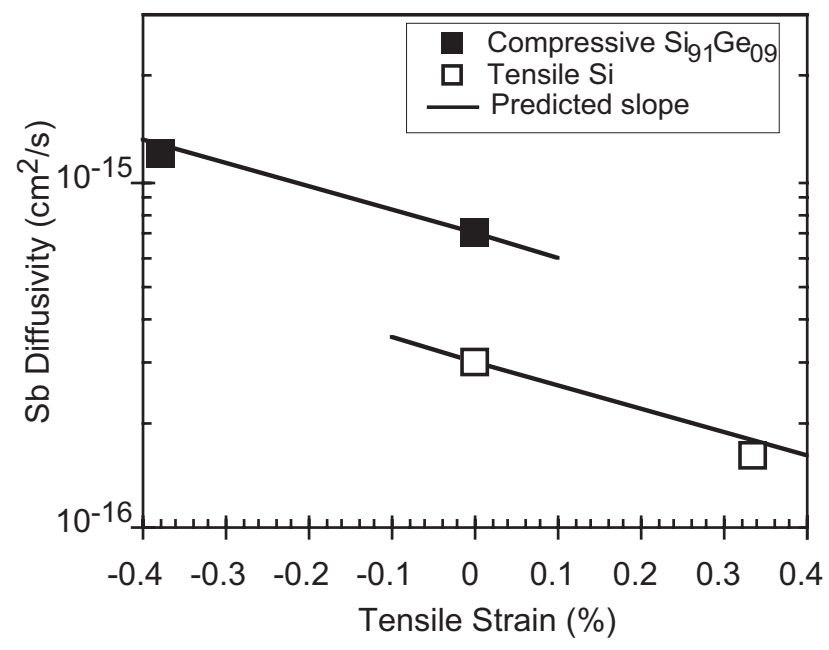

Figure 3. Biaxial strain effect on Sb diffusion in Si-Ge thin film at constant composition. Data points from Kringhoj et al. [5]. The lines have been chosen to go through the data points at zero strain. The slopes of the lines are determined from (14) with no free parameters (zero migration strain anisotropy, as predicted), and indicate the value of $Q^{\prime}$ expected for the biaxial experiment by inputting our measured value of $V^{*}$ under hydrostatic pressure.

The offset between lines is a free parameter representing the effect of composition at constant strain.

Equation (14) with zero migration-strain anisotropy was used [7] to compare the value of $Q^{\prime}$ reported by Kringhoj et al. [5] for $\mathrm{Sb}$ diffusion in biaxially strained $\mathrm{Si}$ and $\mathrm{Si}-\mathrm{Ge}$ and $V^{*}$ measured in hydrostatically compressed $\mathrm{Si}$. The result is $V^{*}+\frac{3}{2} \frac{Q^{\prime}}{Y}=(+0.93 \pm 0.20) \Omega$ for a compressively strained Si91Ge9 alloy and $V^{*}+\frac{3}{2} \frac{Q^{\prime}}{Y}=(+1.20 \pm 0.33) \Omega$ for tensile Si. With no free parameters, these results are in excellent agreement with the prediction of $+1 \Omega$, i.e. zero migrationstrain anisotropy, for a simple vacancy mechanism. Graphically, this agreement is illustrated in Fig. 3, where the biaxial strain effect is predicted from the hydrostatic measurement using this value in (14) and compared to the measured biaxial effect.

A similar analysis has been performed for the existing experiments on $\mathrm{B}$ diffusion in $\mathrm{Si}[4,8,9]$. We extracted values of $Q^{\prime}$ from the measurements of Kuo et al. [4] on the effect of biaxial strain on boron diffusion $\left(D_{33}\right)$ in $\mathrm{Si}_{1-\mathrm{x}} \mathrm{Ge}_{\mathrm{x}}$ films: the solid lines in Fig. 4 are our fits to Kuo et al.'s data with slopes representing $Q^{\prime}=+5.44,-6.01$, and $-5.22 \mathrm{eV}$ per unit strain for $x=0,0.1$, and 0.2 respectively. Using (14) we can interpret the slopes of the solid lines as a "measurement" of the migration strain anisotropy - a determination of the value required to reconcile the observed hydrostatic and biaxial experimental results with an interstitial-based mechanism. The result is $\left(V_{\|}^{m}-V_{\perp}^{m}\right) / \Omega=+1.19,+0.64$, and +0.59 for $x=0,0.1$, and 0.2 respectively ${ }^{4}$. The large values, especially that for pure $\mathrm{Si}$,

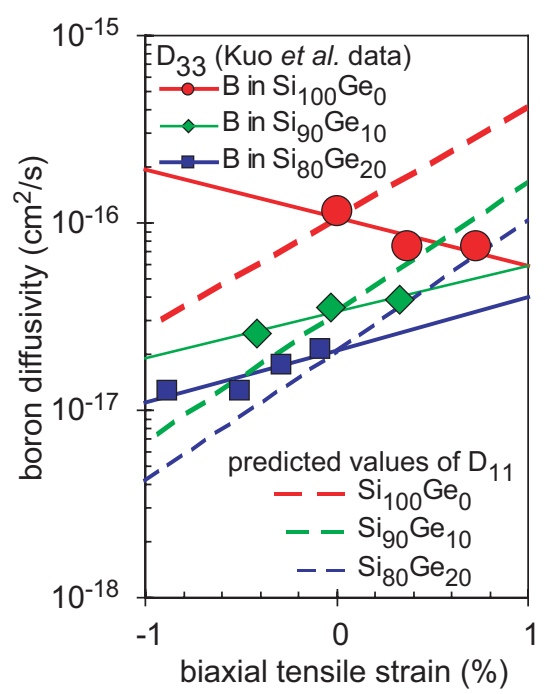

Figure 4. Vertical and predicted lateral diffusivity of B in (001) Si and Si-Ge alloys. Data points are for vertical diffusion in these samples from Kuo et al [4].; solid lines are our fits to obtain $Q^{\prime}$; dashed lines are lateral diffusivities predicted using our measurement of $V^{*}$ and (14).

must be viewed with suspicion. We were concerned about possible artifacts in the Kuo pure $\mathrm{Si}$ data because of the opposite slope of the pure Si data and the alloy data in Fig. 4. We subsequently obtained some of the sample materials grown by Chemical Vapor Deposition (CVD) by Kuo and performed hydrostatic pressure $D_{33}$ measurements on them. Preliminary results indicate an activation volume of $+0.03 \Omega$ for the $10 \%$ Ge alloy and $-0.17 \Omega$ for pure Si. Remarkably, the value for Kuo's pure Si is entirely consistent with the measurements we

\footnotetext{
${ }^{4}$ Here we are making the risky assumption that the activation volume for $x=0.20$ samples, which we have not measured, is identical to that for $x=0.1$. If the mechanism is B-Ge pairing then we might expect any Ge effect to saturate once the Ge concentration exceeded the B concentration.
} 
made $(-0.17 \Omega)$ on samples grown by a different technique (MBE) in a different laboratory. Even more remarkable is the change in $V^{*}$ caused by the presence of just $10 \%$ Ge. Hence our present thinking is that we should take all the experimental results, including Kuo's published values for pure $\mathrm{Si}$, at face value for the moment, see what they might imply, and design experiments to check for consistency among the various experimental and theoretical values and relationships. Additionally, we should view with suspicion any assumption that mechanisms, or numerical values of activation energies or volumes, do not vary significantly with composition. Although there is evidence that the interstitialcy mechanism still predominates in boron diffusion in these alloys [19], it is possible that B-Ge pairing might be responsible for the significant change in $Q^{\prime}$ and $V^{*}$ caused by alloying with Ge, as suggested by Kuo et al. In fact, it appears that as little as $1 \%$ Ge causes a significant $(0.4 \mathrm{eV})$ increase in $E^{*}$ for B diffusion in Si [20]. Further work, both experimental and theoretical, will be required to obtain a consistent and reliable picture.

Because of these nagging concerns about experimental artifacts as well as theoretical over-simplifications, it is very risky to rely on hydrostatic and biaxial $D_{33}$ measurements alone for a measurement of the migration strain anisotropy. A third, independent measurement would be a very valuable consistency test. One such measurement would be the effect of in-plane biaxial stress upon $D_{11}$, the diffusivity in a direction parallel to the surface. From (12) and the analogous equation for $D_{11}$, the migration strain anisotropy can be determined directly from the result of such an experiment:

$$
\frac{D_{11}\left(\sigma_{\text {biax }}\right)}{D_{33}\left(\sigma_{\text {biax }}\right)}=\exp \left[\left(\frac{\sigma_{\text {biax }}}{k T}\right)\left(V_{\|}^{m}-V_{\perp}^{m}\right)\right]
$$

Our predictions for $D_{11}$ from this equation, using values of the migration strain anisotropy inferred from the measurements of $Q^{\prime}$ and $V^{*}$, are shown as the dashed lines in Fig. 4.

\section{PREDICTING ARBITRARY STRESS EFFECTS}

From Eqs. (6) and (8)-(10) it is apparent that for describing diffusion in an (001) wafer, the three parameters $V^{r}, V_{\|}^{m}$ and $V_{\perp}^{m}$ appear in only two independent combinations, $V^{r} / 3+V_{\perp}^{m}$ and $V^{r} / 3+V_{\|}^{m}$. Hence if there is a single defect mechanism and charge state dominating transport then the prediction of the effect of an arbitrary stress state on $D_{33}$ and $D_{11}$ requires (provided the results are reliable) the theoretical determination of the three aforementioned parameters or only two independent measurements (e.g., biaxial and hydrostatic stress effects). If symmetry requires the migration strain anisotropy to be zero then the number is further reduced. In the fabrication of small devices, diffusion in directions other than along the cube axes becomes important. We therefore discuss what further progress is required to permit the prediction of the effect of an arbitrary stress state on diffusion in an arbitrary direction.
The theoretical framework requires some further development. If there are multiple species and charge states involved in diffusion, clearly the contributions from each must be superposed. Additionally, strain breaks the energetic degeneracy of otherwise symmetry-equivalent ground states as well as saddle configurations; in principle they must all be enumerated and superposed [11] but in practice the minimal set of conditions sufficient for zero migration strain anisotropy remains to be seen. Additionally, in a crystal structure whose primitive cell has a multiple-atom basis such as $\mathrm{Si}$, each site in the basis set must be tracked separately [11]. By comparison, the simpler treatment presented in this paper in effect, starting with (8), averages immediately over the symmetry-equivalent configurations, which is normally expected to be reasonable if the degeneracy splitting « $k_{\mathrm{B}} T$ but not otherwise. This treatment is currently the only one that recognizes the non-local nature of the formation strain tensor, which is critical to reconciling the qualitatively opposite effects of hydrostatic compression and of biaxial compression on the diffusivity.

In general the activation strain tensor is a fourth rank tensor; that is,

$$
\ln D_{i j} \propto V_{i j k l}^{*} \sigma_{k l}
$$

where the sum over repeated indices is implied and none of the 81 elements $V_{i j k l}^{*}$ is necessarily zero. Fortunately, often symmetry dictates that only a handful be independent. For a given mechanism (ground state - saddle combination) all of the information about the macroscopic activation strain tensor and its 81 elements is contained within the local configuration change during the transition from the ground state to the saddle configuration. For this transition, the migration strain tensor reckoned from a coordinate system with one axis (say, the third) along the jump direction [11] is given by

$$
\mathbf{\Omega}_{\text {local }}^{\mathbf{m}}=\left[\begin{array}{ccc}
\Omega_{\perp 1}^{\mathrm{m}} & \Omega_{12}^{\mathrm{m}} & \Omega_{13}^{\mathrm{m}} \\
\Omega_{12}^{\mathrm{m}} & \Omega_{\perp 2}^{\mathrm{m}} & \Omega_{23}^{\mathrm{m}} \\
\Omega_{13}^{\mathrm{m}} & \Omega_{23}^{\mathrm{m}} & \Omega_{\|}^{\mathrm{m}}
\end{array}\right],
$$

which has at most six independent elements. Transforming to the crystallographic coordinate system and superposing the contributions for the various defect ground-state and saddlepoint orientations then leads to all elements. If the saddle point configuration is sufficiently symmetric the off-diagonal elements of $\boldsymbol{\Omega}_{\text {local }}^{\mathrm{m}}$ are all zero and the two perpendicular components will be equal to each other, reducing the number of independent elements to two. Note, however, that even in a structure as symmetric as $\mathrm{Si}$, the situation is not this symmetric. The isolated vacancy is believed to have tetragonal Jahn-Teller distortions [21]. The diffusion of donor impurities by a vacancy mechanism is believed to not have a saddle-point configuration in which the impurity has jumped half way into the vacancy, but rather to have one in which the impurity and 
vacancy are at or near third-neighbor sites at opposite ends of a hexagonal ring [22], in which case the off-diagonal elements of $\mathbf{\Omega}_{\text {local }}^{\mathrm{m}}$ are not zero. Some of the outstanding issues discussed above are being addressed in current research.

Once the theoretical formalism is complete, the determination of the handful of elements in the relevant formation strain tensors and in the $\boldsymbol{\Omega}_{\text {local }}^{\mathrm{m}}$ tensors will permit the prediction of the effect of an arbitrary stress state on diffusion in an arbitrary direction. These parameters can be determined by $a b$ initio density functional theory if all of the atoms within the supercell are permitted to relax and the supercell is permitted to undergo arbitrary strains. Certain combinations of these parameters are also observable macroscopically by designing experiments that isolate the effect of certain stress states on diffusion in certain directions. This combination of theory and experiment is a promising area for the testing of theoretical Hamiltonians and methodologies and of models of the relevant diffusion mechanisms. Agreement should be expected so long as the point defect populations remain in equilibrium with the free surface or with some other well-characterized sink. During transient, nonequilibrium processes such as defect reactions and clustering, strain tensors associated with all the kinetic saddle points and metastable species may also be important.

\section{SUMMARY}

Point defect mechanisms are related through thermodynamics to the dependence of the diffusivity on pressure and stress. The influences of hydrostatic and biaxial stress are interdependent in a predictable way for any proposed mechanism. For $\mathrm{Sb}$ in $\mathrm{Si}$, the measured effect of biaxial stress on diffusion can be predicted successfully from the hydrostatic results with no free parameters. For $\mathrm{B}$ in $\mathrm{Si}$, the migration strain anisotropy can be treated as a free parameter and adjusted to reconcile hydrostatic and biaxial results; however nagging concerns remain about the reliability of the resulting picture. Finally, it appears that the means to predict the effect of an arbitrary stress state on diffusion in an arbitrary direction, through experimental and theoretical techniques, are within reach.

\section{ACKNOWLEDGMENTS}

I am grateful to Y. Zhao, H.-J. Gossmann, S. Mitha and D. Schiferl for their collaborations in the experimental work reviewed here; to J.L. Hoyt for providing samples of the CVD material grown by P. Kuo; and to M. Daw and W. Windl for helpful discussions.

\section{REFERENCES}

[1] P.M. Fahey, P.B. Griffin, and J.D. Plummer, Rev. Mod. Phys. 61, 289 (1989).

[2] N. Moriya, L.C. Feldman, H.S. Luftman, C.A. King, J. Bevk, and B. Freer, Phys. Rev. Lett. 71, 883 (1993); F.H. Baumann, J.-H. Huang, J.A. Rentschler, T.Y. Chang, and A. Ourmazd, Phys. Rev. Lett. 73, 448 (1994); N.E.B. Cowern, P.C. Zalm, P. van der Sluis, D.J. Gravesteijn, and W.B. de Boer, Phys. Rev. Lett. 72, 2585 (1994); P. Kuo, J.L. Hoyt, J.F. Gibbons, J.E. Turner, R.D. Jacowitz, and T.I. Kamins, Appl. Phys. Lett. 62, 612 (1993).

[3] N.E.B. Cowern, W.J. Kersten, R.C.M. de Kruif, J.G.M. van Berkum, W.B. de Boer, D.J. Gravesteijn and C.W.T. Bulle-Liewma, in Proc. 4th Int. Symp. on Process Physics and Modeling in Semiconductor Devices, eds. G.R. Srinivasan, C.S. Murthy, and S.T. Dunham, Electrochem. Soc. Proc. Vol. 96-4 (Electrochem. Soc., Pennington, NJ, 1996).

[4] P. Kuo, J.L. Hoyt, J.F. Gibbons, J.E. Turner, and D. Lefforge, Appl. Phys. Lett. 66, 580 (1995).

[5] P. Kringhoj, A. Nylandsted-Larsen, and S.Y. Shirayev, Phys. Rev. Lett. 76, 3372 (1996).

[6] H. Park, K.S. Jones, J.A. Slinkman, and M.E. Law, J. Appl. Phys. 78, 3664 (1995).

[7] M.J. Aziz, Appl. Phys. Lett. 70, 2810 (1997).

[8] M.J. Aziz, Mater. Res. Soc. Symp. Proc. 469, 37 (1997).

[9] Y. Zhao, M.J. Aziz, H.J. Gossmann, S. Mitha, and D. Schiferl, Appl. Phys. Lett. 74, 31 (1999).

[10] Y. Zhao, M.J. Aziz, H.J. Gossmann, S. Mitha, and D. Schiferl, Appl. Phys. Lett. 75, 941 (1999).

[11] M.S. Daw, W. Windl, N.N. Carlson, M. Laudon, and M.P. Masquelier, Phys. Rev. B 64, 045205 (2001).

[12] M. Laudon, N.N. Carlson, M.P. Masquelier, M.S. Daw, and W. Windl, Appl. Phys. Lett. 78, 201 (2001).

[13] G.H. Vineyard, J. Phys. Chem. Solids 3, 121 (1957).

[14] M.J. Aziz, Materials Science in Semiconductor Processing 4, 397 (2001).

[15] F.C. Larche and J.W. Cahn, Acta Metall. 33, 331 (1985); B.J. Spencer, P.W. Voorhees, and S.H. Davis, J. Appl. Phys. 73, 4955 (1993).

[16] P.H. Dederichs and K. Schroeder, Phys. Rev. B 17, 2524 (1978).

[17] M.J. Aziz, P.C. Sabin, and G.-Q. Lu, Phys. Rev. B 44, 9812 (1991).

[18] H.-J. Gossmann, T.E. Haynes, P.A. Stolk, D.C. Jacobson, G.H. Gilmer, J.M. Poate, H.S. Luftman, T.K. Mogi, and M.O. Thompson, Appl. Phys. Lett. 71, 3862 (1997); A. Ural, P.B. Griffin, and J. Plummer, J. Appl. Phys. 85, 6440 (1999).

[19] T.T. Fang, W.T.C. Fang, P.B. Griffin, and J.D. Plummer, Appl. Phys. Lett. 68, 791 (1996).

[20] N.R. Zangenberg, J. Fage-Pedersen, J.L. Hansen, and A. NylandstedLarsen, Defect and Diffusion Forum 194-199, 703 (2001).

[21] A. Antonelli, E. Kaxiras, and D.J. Chadi, Phys. Rev. Lett. 81, 2088 (1998).

[22] S.M. Hu, Phys. Stat. Sol. (b) 60, 595 (1973); O. Sugino and A. Oshiyama, Phys. Rev. B 46, 12335 (1992); J. Xie and S.P. Chen, J. Phys. D 32, 1252 (1999). 